\title{
La policía en el posconflicto: análisis impacto del nuevo Código de Policía y el Escuadrón Móvil Antidisturbios en Colombia
}

\author{
Police in the post-conflict: impact analysis of the new Police Code and the Mobile \\ Riot Squad in Colombia
}

\section{A polícia no pós-conflito: análise impacto do novo Código de Polícia e o Esquadrão Móvel Anti-distúrbios na Colômbia}

\author{
Jheny Karina García Luna $a^{\mathrm{a}^{*}}$ \\ Policía Nacional de Colombia, Bogotá D. C., Colombia
}

- Fecha de recepción: 2019-07-27

- Fecha concepto de evaluación: 2019-09-09

- Fecha de aprobación: 2019-10-28 http://dx.doi.org/ | 0.22335/rlct.v I I i3.986
Para citar este artículo / To reference this article / Para citar este artigo: García, J.K. (2019). La policía en el posconflicto: análisis impacto del nuevo Código de Policía y el Escuadrón Móvil Antidisturbios en Colombia. Revista Logos Ciencia \& Tecnología, I I (3), I07I 19. http://dx.doi.org//0.22335/rlct.vI I i3.986

\section{RESUMEN}

En el marco de la necesidad de comprender para anticipar, teniendo como argumento central que el análisis frente a la intervención del Escuadrón Móvil Antidisturbios (ESMAD) en Colombia en el desarrollo de actividades de protesta social, debe reconocer las dificultades y tensiones que esta tarea representa de manera particular en la estabilización del orden público y orientados en el planteamiento problemático: ¿cuál es el impacto del nuevo Código de Policía y el Escuadrón Móvil Antidisturbios en Colombia en el posconflicto?, se presenta este artículo de abordaje, mediante de la reflexión basada en experiencias internacionales. En este sentido, se propone como objetivo general determinar el impacto del nuevo Código de Policía y el Escuadrón Móvil Antidisturbios en Colombia en el posconflicto, esto bajo una metodología cualitativa y de corte interpretativa, a través de un análisis documental con fuentes primarias y secundarias que permitan identificar las herramientas jurídicas y de actuación vigentes, visibilizando de esta forma la necesidad en materia de gestión de las autoridades políticas administrativas territoriales, en la solución de los requerimientos de la comunidad para evitar el escalamiento del conflicto social y concluyendo la inevitable mutación de problemáticas sociales de todo orden

Palabras clave: policía, orden público, ley, seguridad, código 


\section{SUMMARY}

Within the framework of the need to understand in order to anticipate, having as a central argument the idea that the analysis of the intervention of the Mobile Riot Squadron (ESMAD) in Colombia in the development of social protests, one must recognize the difficulties and tensions that this task represents, particularly in the stabilization of public order and oriented in the problematic approach: What is the impact of the new Police Code and the Mobile Riot Squadron in Colombia in the post-conflict?, this article is presented as a reflection based on international experiences. Therefore, it is proposed, as the general objective, to determine the impact of the new Police Code and the Mobile Anti-Riot Squadron in Colombia on the post-conflict; this under a qualitative and interpretative methodology, through the analysis of documents with primary and secondary sources that allow for the identification of the current legal tools, thus making visible the need for management of territorial administrative political authorities when it comes to solving community requirements in order to avoid the escalation of social conflict and concluding the inevitable mutation of social order problems.

Keywords: police, public order, law, security, code (UNESCO Thesaurus)

\section{SUMÁRIO}

No marco da necessidade de compreender para antecipar, tendo como argumento central que a análise frente à intervenção do Esquadrão Móvel Anti-distúrbios (Esmad) na Colômbia no desenvolvimento de atividades de protesto social deve reconhecer as dificuldades e tensões que esta tarefa representa de maneira particular na estabilização da ordem pública e orientado na abordagem problemática: qual é o impacto do novo Código de Polícia e o Esquadrão Móvel Anti-distúrbios na Colômbia no pós-conflito? Apresenta-se este artigo de abordagem, mediante a reflexão baseada em experiências internacionais. Neste sentido, propõe-se como objetivo geral determinar o impacto do novo Código de Polícia e o Esquadrão Móvel Anti-distúrbios na Colômbia no pós-conflito, isto sob uma metodologia qualitativa e de corte interpretativa, através de uma análise documental com fontes primárias e secundárias que permitam identificar as ferramentas jurídicas e de atuação vigentes, visibilizando desta forma a necessidade em matéria de gestão das autoridades políticas administrativas territoriais, na solução dos requerimentos da comunidade para evitar o escalamento do conflito social e concluindo a inevitável mutação de problemáticas sociais de toda ordem.

Palavras-chave: polícia, ordem pública, lei, segurança, código (Tesauro da UNESCO)

Algunos académicos han señalado el carácter contrainsurgente de la Fuerza Pública en Colombia. "El discurso de la Doctrina de Seguridad Nacional y las políticas heredades de la Guerra Fría, han generado sesgos y estigmatizaciones frente a algunas posiciones políticas, principalmente de izquierda" (Leal, 2003). Lo anterior, ha erosionado la democracia dado el cuestionamiento frente al uso legítimo de la fuerza por parte de las instituciones de seguridad (Cruz, 20I5; Leal, 2003). De ahí que categorías como amenazas a la seguridad o enemigos internos sean señaladas de surgir de un escenario que tradicionalmente ha castigado las expresiones políticas de oposición, normalizando el uso desmedido de la fuerza, bajo un marco institucional aparentemente democrático (Gutiérrez, 20l4).
En Colombia, la protesta social ha sido estigmatizada por largos años. Algunos autores señalan que lo anterior es consecuencia de la asociación de estas expresiones colectivas y los recursos de movilización de las insurgencias. No obstante, se identifica que en Colombia no tiene grandes esfuerzos en este tema, hay muy pocas investigaciones y reflexiones frente este fenómeno.

Se extiende con preocupación que el Gobierno Nacional busca obtener la tranquilidad y el orden público en los escenarios de protesta, preponderando el derecho fundamental. Sin embargo, se han observado diferentes episodios que veremos en este escrito en donde tiene que intervenir la autoridad de policía, para garantizarlo sin limitarlo, todo ello por los malos resultados que las 
protestas han conllevado, dando pie a la intervención del Escuadrón Móvil Antidisturbios (ESMAD) en el desarrollo de las actividades de protesta social.

A través de fuentes primarias y secundarias se observa la necesidad del Estado de regular el derecho a la protesta e identificar un marco normativo que en la actualidad se encuentra en estudio de inexequibilidad, que corresponde a la Ley I80I de 2016, Código Nacional de Policía y Convivencia, para garantizar la protección y garantías de la protesta social, para quienes ejercen la ley haciendo referencia a la autoridad de policía y observando que es el fenómeno de preocupación en un posible escenario de posconflicto.

Reconociendo la importancia de este estudio y el esfuerzo de identificar en la normatividad colombiana la regulación de la protesta, el presente artículo analiza y determina en un escenario de posconflicto la incidencia de la normatividad en los procedimientos de quienes tienen que hacer cumplir la ley, teniendo en cuenta algunos casos de protesta social y la necesidad de la intervención de la Fuerza Pública. Así como el éxito institucional para mitigar el impacto de la protesta por vías de hecho en Colombia, es la articulación de las capacidades para identificar actores promotores y generadores de violencia en la protesta social.

\section{Metodología}

La metodología citada emplea instrumentos cualitativos que permiten obtener información y expone las críticas principales hacia el nuevo Código de Policía y cuáles están directamente relacionadas con las actuaciones del Esmad y la protección a los derechos humanos. Para esta sección se realizaron estudios de fuentes primarias que han estado detrás del seguimiento de esta reforma, bajo una interpretación hermenéutica de la norma y se evaluaron las nuevas demandas de violaciones a los derechos humanos (uso ilegítimo y abusivo de fuerza, tortura, empalamientos, violaciones, uso de armas de fuego convencionales y no convencionales, incluso posibles homicidios en las manifestaciones, basados en CINEP, 20I3) por este organismo que ingresan en el primer año de implementación del código, las cuales son del 2017 y 2018.

Luego, se analizará el nuevo Código de Policía a la luz de la literatura de la teoría de incentivos con el objetivo de identificar las potenciales consecuencias de la nueva normativa en las acciones del ESMAD. Posteriormente, se efectuará un breve análisis de las reformas policiales realizadas en contextos transicionales con el objetivo de identificar las principales lecciones aprendidas. Finalmente, se espera generar una reflexión con recomendaciones y sugerencias al nuevo código, con el fin de garantizar la efectividad de la protección de los derechos humanos en un contexto de posconflicto.

\section{Resultados}

La necesidad de conocer el marco discursivo, la protesta y la movilización social que en ocasiones las relacionan con disturbio, vandalismo o desorden (Cruz, 20 I4, 20 I5; Dudley, 2008; Uprimny \& Vargas, 1990). Lo anterior, ha suscitado el debate sobre la creación de "un marco jurídico punitivo y un modelo de contención policial de la protesta intensivo en fuerza" (Cruz, 20I5, p. 48); en otras palabras, ha sido la represión la forma sistemática de respuesta institucional a las expresiones políticas como la protesta social.

Desde el 2012 se registraron varias protestas sociales de grandes magnitudes en diversas partes del país. Según los datos del Barómetro de las Américas del Latlnamerican Public Opinion Project (LAPOP) (2013), en el 2012 existió un aumento significativo en el porcentaje de ciudadanos que han participado en protestas respecto al 2010, ubicando a Colombia en el quinto país de la región con el mayor porcentaje de participantes en protestas.

El motivo principal para el 2012 estuvo relacionado con la educación, evidenciando el gran impacto de las movilizaciones estudiantiles en contra de la reforma educativa planteada por el Gobierno (Rodríguez \& Seligson, 20I2). Para el 2013, el Centro de Investigación y Educación Popular (CINEP) (20I4) registró I.027 protestas, el número más alto reportado desde 1975 y LAPOP identificó un cambio en los motivos de la participación en protestas sociales con respecto a los años anteriores. Este año los asuntos económicos fueron la principal motivación, lo que demuestra el impacto del Paro Nacional Agrario y otras manifestaciones como la de mineros artesanales, camioneros y estibadores de puertos, las huelgas laborales en empresas multinacionales mineras y petroleras todas estas a nivel regional (LAPOP, 20I3b). Existe evidencia que estos años de auge en la protesta social, estuvieron acompañados de un incremento considerable en las estrategias de contención violenta por las frecuentes acusaciones de la infiltración de la guerrilla, lo cual criminalizó las movili- 
zaciones y generó un escenario de enfrentamiento con la policía (CINEP, 20I4; Cruz, 20I2, 20I3, 2014 y 20I5).

En atención a toda esta problemática de conflictividad social mediante la Resolución 01363 del 14 de abril de 1999, la Policía Nacional de Colombia creó el ESMAD. De acuerdo con la Política Estratégica Operacional y del Servicio de la Policía, el ESMAD, son "unidades policiales especializadas para el manejo y control de multitudes, conformadas por personal capacitado y dotado de los equipos y elementos necesarios para el restablecimiento del orden ciudadano" (Restrepo, 2013). Esta especialidad de la Policía Nacional tiene como objetivo el uso de la fuerza para dispensar masas de personas que incitan 0 practican acciones de violencia y cuenta con la siguiente distribución, según (PONAL, 20I6):

La distribución de los Escuadrones Móviles Antidisturbios se realiza de manera estratégica en aquellos territorios donde permanece el personal disponible para el cumplimiento de su misión, con cobertura en las ocho regiones de policía. En la actualidad se cuenta con 23 móviles de la especialidad antidisturbios conformados por $\mathbf{3 . 5 8 0}$ hombres y mujeres, prestos a velar por el restablecimiento del orden ciudadano cuando este lo requiera, en los Departamentos de Policía Huila, Tolima, Risaralda, Caldas, Valle, Cauca, Nariño, Santander, Magdalena Medio, Córdoba, Antioquia, Meta, Casanare, Atlántico, Cesar, Bolívar, La Guajira y en las Metropolitanas de Policía Bogotá, Cúcuta y Valle de Aburrá.

Este grupo especializado de la Policía es la cara de la institucionalidad en los enfrentamientos con los ciudadanos que participan en las movilizaciones sociales. Desde su creación han existido constantes críticas a su labor y funcionamiento, particularmente por los defensores de derechos humanos, quienes argumentan el uso ilegítimo $y$ abusivo de fuerza, tortura, empalamientos, violaciones, uso de armas de fuego convencionales y no convencionales, incluso posibles homicidios en las manifestaciones (CINEP, 20I3a, 20I3b). Tanto así que las organizaciones civiles y partidos políticos han propuesto el desmonte del ESMAD.

$\mathrm{Al}$ anterior contexto se suma el proceso transicional que estaba viviendo el país tras la firma del Acuerdo de $\mathrm{Paz}$ con las Farc el pasado 24 de noviembre de 2016' (OACP,

I El 2 de octubre de 2017, el Plebiscito tiene como resultado NO, se interpuso con 6.422 .136 votos, el 50,23\% de votos. El Sí reunió un total de 6.361 .762 votos. Permitiendo que fuera posible construir un nuevo acuerdo, que no solo refleja las
2016). Luego de más de sesenta años de conflicto armado interno esta organización se dispone a entregar las armas e iniciar su proceso de desmovilización. En la actualidad, este grupo guerrillero se encuentra en las denominadas Zonas Veredales ${ }^{2}$, las cuales se suspenderán en agosto de 2019 y paralelamente todo el andamiaje está para el mejoramiento de construcción ante mejores condiciones óptimas de implementación ${ }^{3}$. Lo anterior ubica a Colombia en un momento histórico importante, puesto que obliga a todas las instituciones a pensarse en un escenario en ausencia del conflicto armado ${ }^{4}$, los fenómenos futuros en un contexto de seguridad ciudadana

Hoy día se encuentren grupos armados organizados como el Ejército de Liberación Nacional (ELN), Clan del Golfo, Los Pelusos, Caparros y grupos armados organizados residuales (GAO-r); sin embargo, la reingeniería de cara a un posible escenario de posconflicto impacta de manera importante a las instituciones de seguridad, entendida esta seguridad ciudadana desde lo expuesto por Aparicio (2010):

No se basa exclusivamente en el accionar de las agencias de policía o de las organizaciones presentes dentro del sistema penal; ella compromete a todos los ministerios y organizaciones públicas que contribuyen a la convivencia a controlar las causas del delito o formación del individuo (sistema educativo, sistema contravencional, trabajo, asistencia social, etc.); las cuales en conjunto solucionan los problemas de la seguridad ciudadana (p. 24).

Las instituciones de seguridad tendrán que ser garantes del acuerdo y adaptarse a este nuevo escenario. Justamente por lo anterior, este artículo pretende analizar el impacto que se identifica con la reforma al Código de Policía en las acciones y directrices del Esmad, particularmente en la garantía y protección de los derechos humanos por parte de este organismo, teniendo en cuenta que

principales propuestas de los líderes políticos del NO, sino que también ofrece una mejor perspectiva para el posconflicto en Colombia.

2 Contiene varios campamentos de las Farc, donde hay de manera permanente representantes de la ONU.

3 La Comisión para el Esclarecimiento de la Verdad, la Convivencia y la No Repetición tendrá como finalidad que se esclarezca y conozca lo ocurrido en el conflicto, incluyendo las graves violaciones a los derechos humanos e infracciones al Derecho Internacional Humanitario (DIH), hasta el 28 de mayo de 2017, aprobado por el Congreso de la República en marzo de 2017.

4 EI ELN como última guerrilla activa en el país, inició acercamientos en octubre de 2016, instalando mesa de negociación el 7 de febrero de 2017, en la ciudad de QuitoEcuador. 
se estima el pronunciamiento de la Corte Constitucional y que encontramos insuficientes las investigaciones frente este tema, siendo sorprendente no identificar estudios a nivel nacional e internacional, escritos que no relacionan el caso colombiano.

¿Qué cambió y qué siguió igual?: el nuevo Código de Policía

El anterior Código de Policía estaba cobijado bajo el Decreto-Ley 1355 de 1970, es decir, que aproximadamente hace cuarenta y seis años este código no era actualizado, analizado o reformado. La coincidencia entre esta reforma y los momentos de transición que adelante el país hace imperante estudiar la armonía de estas reformas con las expectativas del rol que debe cumplir la policía en periodos de posconflicto.

Por esta razón, el principal objeto del presente artículo es estudiar las actuaciones de un grupo específico de la Policía Nacional frente a la aplicación del Decreto-Ley I 355 de 1970 y la aplicación de la Ley I80 I de 2016 en el 2017, “por la cual se expide el Código Nacional de Policía y Convivencia". Todo ello para definir acciones sostenibles por parte de la Policía Nacional en un posible escenario de posconflicto. Según Niño (20l3):

Hay acciones que deben ser sostenibles por parte de la policía en el posconflicto, como son: seguir combatiendo todas las modalidades de delito, continuar con la articulación del tejido social, la protección integral a la población civil, promover el respeto de los derechos humanos y garantizar la convivencia y la seguridad ciudadana... (p. 5).

La Ley 1355 de 1970, el anterior Código Nacional de Policía, no tenía alcance para enfrentar los nuevos fenómenos que actualmente enfrenta la seguridad y convivencia ciudadana, como tampoco la realidad que vive Colombia después de la Constitución de 1991. Es así que frente a las manifestaciones sociales y lo que hoy llamamos protesta social como garantía constitucional, prescrita en el artículo 37 de la Constitución Política de 1991 en donde se señala: "Toda parte del pueblo puede reunirse y manifestarse pública y pacíficamente. Solo la ley podrá establecer de manera expresa los casos en los cuales se podrá limitar el ejercicio de este derecho". La Ley 1355 de 1970 en ningún de los 230 artículos tiene como contenido el ejercicio de la actividad de la Policía para la protección y garantía de la protesta social en Colombia, como tampoco en las siguientes normas: Decreto Nacional 522 de 197I, sobre contravenciones especiales de competencia y procedimiento de policía; Ley 232 de 1995, "por medio de la cual se dictan normas para el funcionamiento de los establecimientos comerciales"; Ley II 85 de 2008, “ley general de cultura y se dictan otras disposiciones", entre otras, que modificaron y derogaron algunos artículos de este código.

Ahora bien, la Ley 180 I de 2016, nuevo Código Nacional de Policía, no prevé restricciones a las marchas sociales, por el contrario, brinda mayores garantías a la protesta social, mejorando las condiciones establecidas en la actualidad, por ejemplo, se obliga a la intervención de gestores de convivencia civiles antes de cualquier intervención policial (Saavedra, 20I6).

Tal es el caso del capítulo VI en el que prescribe el derecho a la reunión, el cual lo encuentra reglamentado en el artículo 47 de la ley en mención:

Artículo 47. Definición y clasificación de las aglomeraciones de público. Para efectos de las obligaciones relacionadas con el derecho de reunión, entiéndase como aglomeración de público toda reunión de un número plural de personas producto de una convocatoria individual o colectiva. En razón a sus características y requisitos, se establecen tres categorías: I. Reuniones o manifestaciones públicas y pacíficas en el espacio público; 2 . Actividades que involucran aglomeraciones de público no complejas; 3 . Actividades que involucran aglomeraciones de público complejas.

Parágrafo. El Gobierno Nacional determinará, dentro del año siguiente de la expedición de este Código, las variables tales como aforo, tipo de evento, clasificación de edad para el ingreso, lugar donde se desarrolla, infraestructura a utilizar, entorno del lugar, características del público, frecuencia, características de la presentación, carácter de la reunión, que determinarán la clasificación del evento como uno de los señalados en los numerales 2 y 3 del presente artículo (Ley I80I de 2016).

Sin embargo, este artículo actualmente se encuentra declarado inexequible por la Corte Constitucional mediante Sentencia C-223 del 20 de abril de 2017, magistrado ponente: Alberto Rojas Ríos, con efectos diferidos "por un término no mayor al agotamiento de las siguientes legislaturas, es decir, hasta antes del 20 de junio de 2019” (p. 93). 
Cabe resaltar que el títuloVI del Código Nacional de Policía, tiene cuatro capítulos con treinta artículos, que inician desde el artículo 48 y terminan hasta el artículo 75, los cuales regulan toda clase de manifestación; es decir, toda clase de protesta social y la intervención de la Fuerza Pública, en caso de presentarse anomalías en la misma. Situación que no se encontraba regulada en el anterior Código de Policía y en ninguna otra legislación colombiana. Fue justamente la frecuencia de la actividad de protesta en Colombia y la ausencia de una regulación en términos de intervención de la Policía en estas circunstancias, que se justifica altamente la importancia de implementar algún tipo de normatividad. Por ejemplo, desde la aplicación del Código Nacional de Policía, se han presentado 287 actividades de protesta con conflictividad social (bloque, disturbios, tomas y asonadas) evidenciando un incremento del $36 \%$ respecto del mismo período del 2016 donde se presentaron 211 . Siendo el departamento del Valle del Cauca el de mayor número de protestas, registrando un total de 79 actividades (Policía Nacional - DISEC, 2017).

Aparentemente, parece positiva la propuesta del legislativo frente a este tema incluido en el Código Nacional de Policía, pero el número de demandas frente a este articulado indica el inconformismo de algunos sectores de la sociedad.
Entre enero de 2016 a octubre de 2018 , se encontró una identificación de 91 demandas contra esta norma: Ley I80 I de 2016 (Código Nacional de Policía y Convivencia), de las cuales hasta el 2018 solo trece han sido favorables (tal cual como están prescritas en el código objeto de estudio); las demás se encuentran pendientes en fallo, pero hasta enero de 2019 ninguna se ha pronunciado frente los artículos que relacionan las manifestaciones y los cuales hacen referencia a la protesta social (Policía Nacional - Secretaría General, 20I7).

Aun cuando el presente artículo estudia las inexequibilidades frente a la regulación de la protesta que dictaminó el Código de Policía, en la figura I se presenta un esquema para exponer las inexequibilidades de la Ley I80I de 2016 (Código Nacional de Policía y Convivencia).

Frente las demandas realizadas a este articulado, se identifica una inexequibilidad diferida por un término de dos años, $y$ una inexequibilidad inmediata al artículo 48 que reglamenta el derecho a la reunión y el artículo 162, señalaba algunas facultades a la autoridad para ingresar al inmueble. En consecuencia, el Código Nacional de Policía tenía hasta el 2018 en proceso treinta y una demandas en contra, de las cuales la Corte Constitucional ha fallado cuatro demandas (ha decretado dos artículos exequibles y tres inexequibles), dejando a la Policía Nacional, del tí-

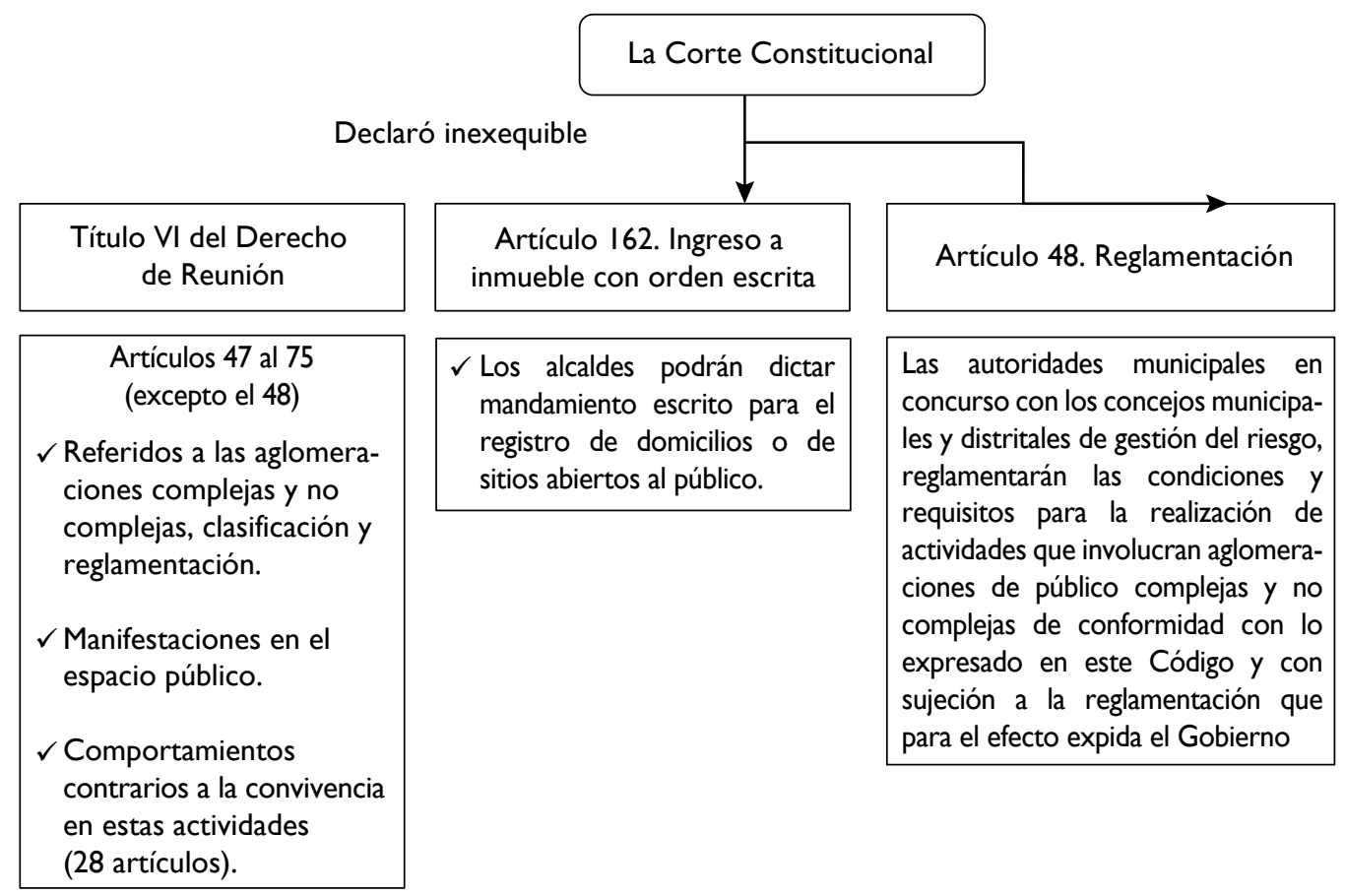

Figura I. Inexequibilidades Ley I80I de 2016 
tulo VI, la aplicación únicamente de veintiocho artículos frente al derecho de reunión, hasta que el Congreso emita la ley estatutaria que reglamente este derecho (Policía Nacional - Secretaría General, 2017).

\section{Marco internacional}

Es importante el papel que puede desempeñar la comunicación entre los manifestantes, las autoridades locales y policía, en la gestión adecuada de concentraciones, en las manifestaciones pacíficas (ONU, 20l4). Es así que países como Argentina, Chile, España, Grecia, Egipto, México, Corea del Sur, Panamá, Francia, Alemania, Australia, cuentan con policías antidisturbios, los cuales, mediante imágenes transmitidas por los medios comunicación, tienen fuertes críticos por el uso de la fuerza, pues su fin último es controlar el accionar de multitudes, evitando que generen daños a terceros.

Existe literatura que orienta el tratamiento asertivo a la manifestación de la protesta social, esto bajo el criterio de su naturaleza pacífica. Desde esta perspectiva, se relaciona la publicación conjunta de la Oficina Regional para América del Sur del Alto Comisionado de las Naciones Unidas para los Derechos Humanos (ACNUDH) y el Instituto Nacional de Derechos Humanos de Chile (INDH), denominada Protesta Social y Derechos Humanos: Estándares Internacionales y Nacionales, en esta plantean estándares aplicables al ejercicio de la protesta social.

De igual manera, existen autores que realizan ejercicios académicos que orientan reflexiones al respecto a partir de constructos teóricos. Por ejemplo: teorías de la atribución (método para evaluar cómo la gente explica el origen de su propio comportamiento y el de los demás (Rodríguez, 2018); teoría de la frustración/agresión (Bandura \& Walters, 1963), estos autores exponen que la agresión es siempre consecuencia directa de una frustración previa, y a la inversa, la existencia de una frustración siempre conduce a alguna forma de agresión; psicología de masas (Moscovice, citado por Fernández, 20I2): una masa es un conjunto transitorio de individuos iguales, anónimos y semejantes, en el seno del cual las ideas y las emociones de cada uno tienden a expresarse espontáneamente; entre otros. Desde esta perspectiva, es necesario el abordaje con elementos constituidos académicamente por la dinámica de estos escenarios, como expone Guillén (2019):

Las dinámicas de estas concentraciones humanas son muy complejas, ya que concurren muchos actores con intereses y objetivos diversos, lo que puede propiciar algún incidente que detone un estallido de violencia y ponga en crisis la paz social y política. Es por ello [por lo] que, desde disciplinas diversas (psicología, sociología, criminología), se han llevado a cabo estudios para conocer el funcionamiento de las masas y cuáles son las pautas de actuación que las autoridades y, muy concretamente, la Policía tendría que seguir para conseguir que los eventos correspondientes se lleven a cabo, ya que los ciudadanos lo hacen en el legítimo ejercicio de sus derechos de manera pacífica sostenible posible.

Así las cosas, como referente principal internacional frente las protestas tenemos a España donde se observa la intervención de la policía antidisturbios, con especial hincapié por las manifestaciones problemáticas; es así que se intenta describir cómo se realiza en este país la gestión de las protestas públicas. Para ello es necesario conocer cuántas tienen lugar, qué tipo de incidentes ocurren durante las mismas y cómo se responde a ellos. Con una aproximación a los datos oficiales publicados por el Ministerio del Interior. Aunque estos datos cuantitativos no son suficientes para conocer las distintas dimensiones de la policía del orden público (McPhail, Schweingruber \& McCarthy, 1998) sí resulta un punto de partida necesario.

Una primera forma de abordar el control de protestas en España consiste en acudir a las estadísticas que publica de forma periódica el Ministerio del Interior en sus anuarios (1998-20II $)$, bajo el epígrafe Ejercicio de los derechos fundamentales (Blay, 2013) y el subepígrafe Derecho de reunión y manifestación (Blay, 20I3).

En la tabla I puede observarse que con el tiempo la tendencia en las manifestaciones presentó un aumento en el número total y que conforme aumenta el porcentaje de ellas es inmediatamente comunicado a las autoridades evidenciando una intervención policial, específicamente por las no comunicadas a las autoridades gubernamentales.

Ante este número tan amplio de manifestaciones, el Ministerio del Interior para el cual forma parte la Policía Antidisturbios de España, refleja anualmente las estadísticas acerca de las detenciones, actuaciones de la institucionalidad, daños a instalaciones, coacciones y retenciones, heridos, barricadas, encadenamientos, ocupación de loca-

\footnotetext{
5 El periodo reflejado, 1998-20II responde a los Anuarios Estadísticos disponibles en la página web del Ministerio del Interior, http://www.interior.gob.es/anuariosestadisticos-12/anuario-estadistico-de-201 I-1892.
} 
Tabla I. Manifestaciones (comunicadas, no comunicadas y prohibidas)

\begin{tabular}{|c|c|c|c|c|c|c|c|}
\hline & \multicolumn{2}{|c|}{ Comunicadas } & \multicolumn{2}{|c|}{ No comunicadas } & \multicolumn{2}{|c|}{ Prohibidas } & \multirow{2}{*}{$\begin{array}{c}\text { Total } \\
\text { Número }\end{array}$} \\
\hline & Número & Porcentaje & Número & Porcentaje & Número & Porcentaje & \\
\hline 1998 & 3.469 & $33,88 \%$ & 6.769 & $66,12 \%$ & 3 & - & 10.238 \\
\hline 1999 & 3.318 & $33,92 \%$ & 6.465 & $66,08 \%$ & I & - & 9.783 \\
\hline 2000 & 4.034 & $32,80 \%$ & 8.264 & $67,20 \%$ & 39 & - & 12.298 \\
\hline 2001 & 4.790 & - & 7.457 & - & 50 & - & 12.247 \\
\hline 2002 & & - & - & - & - & - & \\
\hline 2003 & 4.088 & $41,48 \%$ & 5.766 & $58,52 \%$ & 106 & $1,07 \%$ & 9.854 \\
\hline 2004 & 3.737 & $46,75 \%$ & 4.255 & $53,24 \%$ & 28 & $0,35 \%$ & 7.992 \\
\hline 2005 & 3.789 & $55,53 \%$ & 2.985 & $44,06 \%$ & 7 & n.d. & 6.774 \\
\hline 2006 & 4.096 & $44,04 \%$ & 5.165 & $65,94 \%$ & 2 & n.d. & 9.231 \\
\hline 2007 & 4.527 & $49,57 \%$ & 4.604 & $50,43 \%$ & 12 & - & 9.131 \\
\hline 2008 & 8.760 & $57,53 \%$ & 6.466 & $42,47 \%$ & 139 & $0,91 \%$ & 15.226 \\
\hline 2009 & 18.568 & $76,34 \%$ & 5.455 & $22,43 \%$ & 297 & $1,22 \%$ & 24.023 \\
\hline 2010 & 21.941 & - & - & - & 273 & - & 22.214 \\
\hline 2011 & 21.297 & - & - & - & 371 & - & 21.668 \\
\hline
\end{tabular}

Fuente: Blay (2012), partir de datos del Ministerio del Interior (Anuarios 1998-20II).

les, encierros, cortes de tráfico y cortes de vías férreas que es el resultado final de estas actuaciones (Blay, 20I3).

Al verificarse los datos y al compararse las cifras de las manifestaciones $y$, en especial, sobre incidencias que tienen los medios de comunicación y la actuación de la policía con datos sobre Cataluña (Guillén, 20I2), se encuentra que la mayoría de las manifestaciones transcurren de forma pacífica y en la mayoría de los casos la tarea de la policía se limita a garantizar el orden público para facilitar el ejercicio del derecho de reunión y manifestación de los que protestan. De las observaciones anteriores surgen dudas suficientes que invitan a buscar fuentes alternativas de datos para verificar la fiabilidad de los datos oficiales y para poder realizar una aproximación más informada al modelo de control de protestas.

En este marco internacional podemos inducir que el modelo policial de control de protestas de diferentes países, específicamente en España, tendría para la sociedad, una mayoría de las protestas con modelo de gestión negociada por parte de las autoridades, quien represente $o$ haga sus veces como autoridad. Sin embargo, dentro de las exposiciones y las diversas consideraciones en donde se afirme que efectivamente se evidencien los casos de protestas transgresoras, se considera que no hay uno mejor, sino que este depende de las variadas circunstancias, esto bajo el modelo de incapacitación selectiva con los estándares internacionales para el uso de la fuerza (Gillham \& Noakes, 2007).

Se evidencian dos tendencias distintas para enfrentar las manifestaciones sociales, con la facilitación y negociación de protestas tradicionales o contenidas, en la cual la intervención de la policía es de orientación y de estrategias más duras para las protestas transgresoras (Blay, 2013), donde el actuar policial se extiende a las acciones pertinentes que normalicen el entorno social. No obstante, este tipo de estrategias descritas tienden a demarcar cómo los ciudadanos expresan sus opiniones políticas y sitúan en la policía el poder de configurar el campo de la protesta mediante intervenciones unilaterales y si es necesario coactivas (Della Porta, Peterson \& Reiter, 2006).

Las dinámicas de movilizaciones como la Primavera Árabe en el 2010 y en Brasil durante la copa confederaciones por inconformismos sociales, surgieron como una de las más importantes representaciones del siglo XXI para la reivindicación de necesidades insatisfechas por la sociedad a nivel mundial.

Los medios sociales se han constituido como dinamizadores de la globalización y su impacto como factor de éxito de grandes movilizaciones, evidenciando resultados de trascendencia a favor de los manifestantes. 
El eje gravitacional de las convocatorias se potencializó a partir de la masificación de los medios sociales (WhatsApp, Facebook, Twitter), como el principal instrumento de articulación y método de convocatoria, garantizando difusiones masivas, minimizando costos en la convocatoria, vinculando sectores no tradicionales y el anonimato de sus promotores.

En Colombia, este modelo empezó a evidenciarse con cualidades diferenciales desde el 2013 , siendo el punto de partida de nuevas tendencias de movilización, mejorando la cohesión de organizaciones sociales, especialización de los métodos para el desarrollo de actividades de protesta violenta y sostenida.

Esta situación generó un efecto denominado "bola de nieve", donde se vincularon diversos sectores, los cuales protestaron desde su territorio, motivando una articulación multisectorial con el mismo objetivo (paralizar el país) pero con diferentes intereses, los cuales se materializaron en un Paro Nacional Agrario con una duración de 52 días, nunca visto en la historia de Colombia.

A raíz de esto, y de todo la aplicación de estos casos en materia internacional, puede evidenciarse que Colombia sin transgredir los derechos humanos y la Constitución, tiene la obligación de crear una norma y grupos especiales que puedan estabilizar el orden público; lo anterior teniendo en cuenta que a través de este estudio se identificó un incremento progresivo y generalizado de actividades de protesta, de otros sectores que identificaron posibilidades de solución de peticiones anteriores.

\section{Discusión}

¿Cuáles son las principales críticas con respecto a las actuaciones del Escuadrón MóvilAntidisturbios?

Antes y después del Código

Desde el 30 de enero de 2017 se observa un aumento de críticas frente a las actuaciones del Esmad, pues el derecho a la protesta en el nuevo código sitúa a la Fuerza Policial en preceptos constitucionales para actuar en caso de disturbios, bloqueos, vandalismo y demás hechos que según el Código Nacional de Policía pueda afectar la seguridad, convivencia y tranquilidad ciudadana.

A continuación, se exponen algunos de los pronunciamientos presentados frente al proceder del Esmad, ante la aplicación de la normatividad del Código Nacional de Policía:
Ramos (2016) es integrante de la Comisión de Paz de la Organización Social y Política del Congreso de los Pueblos y son ellos quienes hacen un análisis del derecho a la protesta e indican:

Sin embargo, la doctrina de seguridad que sigue teniendo como ejes la lucha contrainsurgente $y$ el silenciamiento de la oposición, la reciente ley de seguridad ciudadana, la existencia del Esmad, la normatividad penal y este Código de Policía, son en la práctica los instrumentos de represión legal del Establecimiento, sumados por supuesto a la represión paramilitar que sigue actuando en total impunidad y connivencia con la fuerza pública.

Quinto: otro principio que se vulnera, pese a estar nominalmente incluido en el Código, es el principio de proporcionalidad, razonabilidad y ultima ratio para el uso de la fuerza, así como el carácter de mínima intervención a través de normas y sanciones con carácter punitivo y restrictivo de la libertad (p. I).

Incluso, antes de la promulgación del Código Nacional de Policía, Cruz (2015) indica que en diferentes cifras globales del periodo de protesta social entre el II de junio y el 7 de septiembre de 2013 (Paro Agrario), la policía fue presuntamente responsable de algunos hechos de violación a los derechos humanos (uso ilegítimo y abusivo de fuerza, tortura, empalamientos, violaciones, uso de armas de fuego convencionales y no convencionales, incluso posibles homicidios en las manifestaciones, basados en CINEP, 20I $3 \mathrm{a}$, siendo el $52 \%$ por el ESMAD).

Toda esta situación lleva a algunos congresistas, al Ministro de Defensa y al Director de la Policía Nacional a rendir cuentas en debates de control político por el presunto exceso de fuerza en el paro Agrario, planteando de esta forma la necesidad de desmontar o reformar el Esmad (Cruz, 20I5).

Estos pronunciamientos hacen evidente el debate actual sobre los potenciales impactos del nuevo Código de Policía en las actuaciones del Esmad. Particularmente, sus facultades y afectaciones sobre los derechos humanos en el marco de las protestas sociales.

Con el propósito de entender los puntos en tensión más importantes en el Código y las justificaciones que presentan quienes lo diseñaron, a continuación, se presentan algunas reflexiones producto de entrevistas realizadas a 
críticos de la reforma y algunos miembros de la Policía que participaron en el diseño del Código de Policía.

El primer desafío institucional en materia de protesta social, inició con la atención de actividades regionales de interés particular, que promovió el surgimiento de nuevas organizaciones que exigían atención a sus necesidades.

El Gobierno Nacional en procura de garantizar los derechos y libertades públicas ha encaminado sus estrategias para realizar propuestas para la atención de esta problemática, generando alternativas para la atención de la conflictividad social en el ámbito nacional y para ello por medio de la Policía Nacional se identifica un grupo especial denominado Escuadrón Móvil Antidisturbios.

La coordinación integral y dinámica de un equipo de trabajo permitió establecer que, en escenarios de posconflicto, hay que tener alternativas temáticas y propositivas frente a la solución, evolución y seguimiento de problemáticas reales y su incidencia en la seguridad y convivencia ciudadana.

El manejo de crisis ha permitido la profesionalización de los encargados del control de multitudes, lograr ser un referente como fuerza efectiva para mitigar el impacto de la protesta.

Con la implementación del Escuadrón Móvil Antidisturbios en 1999, se han logrado cambios doctrinales en el control de multitudes; la inclusión de la mujer policía en estos grupos también ha generado un acercamiento a la comunidad femenina en el marco de la manifestación, situación que ha sido referente, en el entendido que no es observado en comunidades internacionales; así como la adopción de nuevas tecnologías y métodos logrando un reconocimiento internacional de Colombia en el control de multitudes por parte de grupos especiales de instituciones del Estado, ya reconocido como estampilla que conjuga las $3 \mathrm{E}$ (eficacia, eficiencia y efectividad).

Desde el punto de vista de la normatividad, identificamos que en Colombia la Constitución Política regula la protesta social como un derecho. No obstante, este estudio ha permitido identificar que en el desarrollo del mismo ha transgredido la seguridad ciudadana, la paz y tranquilidad de los ciudadanos de algunas regiones en el desarrollo de la misma, trayendo a correlación la región del Cauca, Huila, Tolima en el Paro Nacional Agrario. Por consiguiente, el Código Nacional de Policía se encuentra como única norma para la regulación de la misma; no obstante, la inexequibilidad de la norma y la no creación de una ley estatutaria permiten observar la debilidad del Estado para el desarrollo de estas actividades.

Finalmente, se prevé que bajo los resultados de experiencias internacionales se tengan en cuenta los siguientes aspectos:

a) La necesidad de buscar la gestión con las entidades que tienen responsabilidad en la solución de las problemáticas y la definición de los roles para la atención de los escenarios de protesta, se observa que en Colombia el no seguimiento de los compromisos del Gobierno con las organizaciones sociales, sindicales y gremios generan el aumento de inconformismo.

b) Determinar la necesidad fundamental que, desde las autoridades políticas administrativas de las regiones, se comprometan a tomar parte activa en la solución de los requerimientos de la comunidad para evitar el escalamiento del conflicto social, estos cumplimientos deben ser públicos y conocidos por los medios de comunicación.

c) Mejorar algunas instancias de coordinación a nivel interno que permitan a través de las lecciones internacionales como México, Chile y España, fortalecer de forma transversal la capacidad del Gobierno Nacional en la atención a las protestas, capacitando a la Fuerza Pública frente a experiencias internacionales ante esta problemática social.

d) Visibilizar de manera proactiva las actividades que realiza el Estado para que la comunidad tenga un concepto favorable de legitimidad en el actuar del Gobierno con las instituciones frente al desarrollo a la protesta social.

e) Regular la protesta social en Colombia con aprobación de las Naciones Unidas, dejando entrever que no es limitar un derecho fundamental, sino garantizarlo mediante una ley con estudio de constitucionalidad, es decir una ley estatutaria.

\section{Conclusiones}

Llegando a escenarios de posconflicto donde se prevé la terminación de un conflicto armado, se concluye la mutación de otros problemas sociales transformados en conflictos sociales, los cuales son el desacuerdo y la oposición colectiva que surgen en diversos grupos, organizaciones que sienten que sus necesidades básicas no están siendo satisfechas y toman el derecho a la protesta social como el único mecanismo para realizar sus manifestaciones frente al desacuerdo del Gobierno Nacional; resalta que los actores buscan presionar la solución de las problemáticas a través de acciones (bloqueos o disturbios) que van en contravía de la ley y que por su variabilidad es compleja la gestión institucional. 
Ya que las posibilidades de que se produzca algún hecho improvisto que desencadene en violencia, no son menospreciables. Es decir, la presencia de policías, representantes de partidos políticos, sindicatos, ONG, periodistas y público en un mismo espacio y posicionándose sobre temas muy espinosos, constituye un coctel que no es fácil de preparar ni de digerir (Guillén, 2019).

Por ello, debe tenerse presente: ¿cuál es la función de la Policía Nacional frente a la protesta social?, en el estudio, observamos que la Policía Nacional como garante de la seguridad ciudadana contextualizó la identificación de escenarios de conflictividad social, los cuales se presentaron en época de conflicto armado, en etapa de diálogos con las Farc y que actualmente puede afectar la paz y la tranquilidad de los colombianos con el mal control y regulación de las protestas sociales.

En cuanto a temas normativos y doctrinales, se expuso el documento que expidió el Ministerio del Interior (Resolución II 90 de 2018), por la cual se adopta el protocolo para la coordinación de las acciones de respeto y garantía a la protesta pacífica que establece las acciones de la Policía Nacional en el desarrollo de la movilización pacífica, que se basa en garantizar el ejercicio de los derechos y libertades públicas tanto de manifestantes como de aquellos que no participan de la manifestación.

Se hace necesario observar: ¿cuáles son los actores que intervienen en las protestas? A través del presente estudio identificado en las demandas realizadas contra la normatividad de la protesta social y la inexequibilidad de las mismas, se determinó que hay tres actores involucrados en estas actividades: I) grupos de manifestantes (estudiantes, sindicatos, indígenas, problemáticas emergentes, entre otros), 2) autoridades competentes (presidente, ministros, gobernadores, alcaldes, entre otros) y 3) Policía Nacional. Esto demanda la correcta articulación entre actores, toda vez que, aún con los roles que cada uno adquiere, la intervención desproporcionada (especialmente de la Policía) puede violentar las expresiones de manifestación pacífica.

Ahora bien, para ello la construcción de propuestas de mejoramiento frente esta problemática de posibles escenarios de posconflicto, deben tenerse bajo un marco normativo que en Colombia no existe y que con la inexequibilidad de los artículos que refieren este tema en el Código Nacional de Policía y de Convivencia (Ley I80 I de
2016), el legislador tendría que proponer una ley estatuaria que regule la protesta social, la cual no existe.

\section{Agradecimientos}

Gratitud a la doctora Alejandra Ortiz Ayala, docente de la Universidad de Nuestra Señora del Rosario; a la Policía Nacional quien contribuyó con información para el presente escrito, el apoyo y orientación fue fundamental en el presente escrito.

\section{Reconocimientos}

El presente artículo es producto de la Maestría en Derecho con énfasis en Derechos Humanos y Justicia Transicional de la Universidad Colegio Mayor de Nuestra Señora del Rosario

\section{Referencias}

Aparicio, J. (2019). De la seguridad nacional a la seguridad ciudadana: el papel de la Policía Nacional. Bogotá: Policía Nacional.

Bandura, A. y Walters, R. (1963). Aprendizaje social y desarrollo de la personalidad. Madrid:Alianza Editorial.

Blay, E. (2013). El control policial de las protestas. InDret Revista para el Análisis de Derecho, 4, I-32.

Centro de Investigación y Educación Popular (CINEP). (2013a). Programa por la paz. Luchas sociales, derechos humanos y representación política del campesinado I988-20 I 2 [informe especial]. Bogotá: CINEP.

Centro de Investigación y Educación Popular (CINEP). (20I3b). Panorama nacional de derechos humanos y violencia política en Colombia. Banco de datos de violencia política. Revista Noche y Niebla, 47, I-288.

Centro de Investigación y Educación Popular (CINEP). (2014). Luchas sociales en Colombia 2013. Bogotá: CINEP.

Corte Constitucional de Colombia - Sala Plena. C-223/I7. Referencia: expedientes D-II604 y D-II6II. Demanda de inconstitucionalidad contra los artículos 47, 48, 53 (parcial), 54, 55 y 162 de la Ley I80I de 2016. Magistrado ponente: Alberto Rojas Ríos (20 de abril de 2017).

Cruz, E. (2012). La MANE y el paro nacional universitario de 201 I en Colombia. Ciencia Política, I4, I40-193.

Cruz, E. (20I3).Todos somos hijos del café: sociología política del paro nacional cafetero. Entramado, 9(2), I38-I58. 
Cruz, E. (20l4). Dignidad en movimiento. El ascenso de la movilización social en Colombia. Confluenze. Revista di Studi Iberoamericani Università di Bologna, 6(25), 24I-275.

Cruz, E. (20I5). El derecho a la protesta social en Colombia. Pensamiento Jurídico, 42, 47-69. Recuperado de https://revistas.unal.edu.co/index.php/peju/article/view/55404.

Della-Porta, D., Peterson, A., \& Reiter, H. (2006). The policing transnational protests. En D. Della-Porta, A. Peterson y $\mathrm{H}$. Reiter (Eds). Introduction. The policing of transnational protest (pp. I-12). Burlington,VT:Ashgat.

Dudley, S. (2008). Armas y urnas. Bogotá: Planeta.

Fernández,A. M. (20I2). Psicología de masas, identidad social, epidemias y rumores: la influenza en México. Sociológica, 230.

Guillén, F. (20I2). Policia i seguretat. Barcelona: Universidad Autónoma de Barcelona.

Guillén, F. (2019). El orden público en las grandes concentraciones de personas. Dinámicas y líneas de intervención. En M. Siller y D. G. Hernández, Estudios de violencia (p. 294). Ciudad de México, México: Editorial Flores.

Gillham, P. F., \& Noakes, J. A. (2007). More than a march in a circle:Transgressive protests and the limits of negotiated management. Mobilization, I2(4), 34 I-357.

Gutiérrez, F. (20|4). Estructura organizacional de los paramilitares y derechos de propiedad en el campo (1982-2007). Análisis Político, 82, 3-2I. Recuperado de http://www.observatoriodetierras.org/wp-content/uploads/2015/08/ Guti\%C3\%A9rrez-20I4-Paola-y-Vargas-20I4.pdf.

Latin American Public Opinion Project (LAPOP) (2013a). Barómetro de las Américas. Nashville: AmericasBarometer. org. Recuperado de https://www.vanderbilt.edu/lapop/ LAPOPI21814_Spanish.pdf.

Latin American Public Opinion Project (LAPOP). (2013b). Cultura política de la democracia en Colombia 20 I3: actitudes democráticas en el contexto del proceso de paz. Bogotá: Universidad de los Andes, Vanderbilt University.

Leal, F. (2003). La doctrina de seguridad nacional: materialización de la guerra fría en América del Sur. Revista de Estudios Sociales, 15, 74-87.

McPhail, C., Schweingruber, S., \& McCarthy, J. (1998). Policing protest int he US: 1960-1995. Social Movements, Protest, and Contention, 6, 49-69.

Ministerio del Interior de España. (2012). Anuario estadístico de 1998-20II. Recuperado de http://www.interior. gob.es/anuarios-estadisticos- I 2/anuario-estadisticode-20 I I - I 892\%20[\%C3\%BAltima\%20consulta\%20 26/09/2013].
Niño, J. I. (20I3). El rol de la Policía Nacional de Colombia en el postconflicto: análisis de once experiencias internacionales. Bogotá: Dirección Nacional de Escuelas de Postgrado de Policía.

Oficina Alto Comisionado para la Paz - OACP (2016). Acuerdo final para la terminación del conflicto y la construcción de una paz estable y duradera. Recuperado de http://www. altocomisionadoparalapaz.gov.co/Documents/bibliotecaproceso-paz-farc/proceso-paz-farc-acuerdo-final.pdf.

ONU. (20/4). Protesta Social y Derechos Humanos: Estándares Internacionales y Nacionales. Santiago de Chile: ACNUDH- INDH.

Policía Nacional de Colombia - Dirección de Seguridad Ciudadana (DISEC-PONAL). (2017). Manifestaciones en 20 /6-2 197. Bogotá: DISEC-PONAL. Base de datos.

Policía Nacional - Secretaría General (2017). Inexequibilidades de la Ley I80I de 2016, Código Nacional de Policía y Convivencia. Bogotá: SEGEN-PONAL. Base de datos.

Policía Nacional de Colombia (PONAL). (2016). Policía Nacional de Colombia. Recuperado de https://www.policia.gov. co/espcializados/antidisturbios/distribucion.

Ramos, P. (2016). [Análisis] Derecho a la protesta en el nuevo Código de Policía: ¿en contravía de la paz? Colombia Informa. Sección Política. Recuperado de http://www.colombiainforma.info/analisis-derecho-a-la-protesta-en-elnuevo-codigo-de-policia-en-contravia-de-la-paz/.

Restrepo, J. D. (20I3). ¿Qué hacer con el Esmad? Revista Semana. Sección Opinión. Recuperado de https://www.semana.com/opinion/articulo/que-hacer-esmad/351678-3.

Rodríguez, C., \& Seligson, M. (20I2). Cultura política de la democracia en Colombia y en las Américas, 2012 hacia la igualdad de oportunidades. Observatorio de la democracia. Bogotá: USAID, Universidad de los Andes, Observatorio de la democracia, Centro Nacional de Consultoría LAPOP y Vanderbilt University.

Rodríguez, E. M. (2018). Cómo explicamos el comportamiento: la teoría de la atribución. Recuperado de https://lamenteesmaravillosa.com/explicamos-comportamientola-teoria-la-atribucion/.

Saavedra, A. M. (5 junio, 2016). ¿Cuáles son los puntos polémicos del nuevo Código de Policía? Diario El País. Editorial. Recuperado de https://www.elpais.com.co/judicial/ cuales-son-los-puntos-polemicos-del-nuevo-codigo-depolicia.html.

Uprimny, R., \& Vargas, A. (1990). La palabra y la sangre: violencia, legalidad y guerra sucia en Colombia. En G. Palacio (Comp.). La irrupción del paraestado. Ensayos sobre la crisis colombiana. Bogotá: ILSA, Cerec. 


\section{Legislación}

Constitución Política de Colombia de 1991. Revisada y actualizada. Bogotá: Corte Constitucional. Recuperado de http://www.corteconstitucional.gov.co/inicio/Constitucion\%20politica\%20de\%20Colombia.pdf.

Decreto 1355 de 1970, por el cual se dictan normas sobre policía. Bogotá: Presidencia de la República de Colombia. Diario Oficial 30.139 (2 de septiembre de 1970).

Decreto Nacional 522 de 197I, por el cual se restablece la vigencia de algunos artículos del Código Penal, se definen como delitos determinados hechos considerados hoy como contravenciones, se incorporan al Decreto-Ley I 355 de 4 de agosto de 1970 determinadas contravenciones y se determina su competencia y procedimiento, se modifican y derogan algunas de las disposiciones de dicho decreto, se deroga el Decreto-Ley 1 I 18 de 15 de julio de 1970 y se dictan otras disposiciones. Bogotá: República de Colombia. Diario Oficial 33.300 (29 de abril de 197I).

Ley 232 de 1995, por la cual se dictan normas para el funcionamiento de los establecimientos comerciales. Bogotá: Congreso de Colombia. Diario Oficial 42.162 (26 de diciembre de 1995).
Ley 1185 de 2008, por la cual se modifica y adiciona la Ley 397 de 1997 — Ley General de Cultura-y se dictan otras disposiciones. Bogotá: Congreso de Colombia. Diario Oficial 46.929 ( 12 de marzo de 2008).

Ley I80I de 2016, por la cual se expide el Código Nacional de Policía y Convivencia. Bogotá: Congreso de Colombia. Diario Oficial 49.948 (29 de julio de 2016).

Resolución 01363 del I4 de abril de 1999, por la cual se crea el Escuadrón Móvil Antidisturbios de la Policía Nacional. Bogotá: Polinal.

Resolución II 90 del 2018. Por la cual se adopta el Protocolo para la coordinación de las acciones de respeto y garantía a la protesta pacífica como un ejercicio legítimo de los derechos de reunión, manifestación pública y pacífica, libertad de asociación, libre de circulación, a la libre expresión, libertad de conciencia, a la oposición y a la participación, inclusive de quienes no participan en la protesta pacífica. Bogotá: Ministerio del Interior. Recuperado de https://www.mininterior.gov.co/sites/default/files/resol1 190-18-adopta_protocolo_protesta_pacifica.pdf. 